\title{
Numerical Studies on Time Variations of the Troposphere-Stratosphere Coupled System
}

\author{
Shigeo YODEN, Masakazu TAGUCHI, and Yoko NAITO \\ Department of Geophysics, Kyoto University, Kyoto, Japan
}

(Manuscript received 28 May 2001, in revised form 13 April 2002)

\begin{abstract}
Interannual variation is a year-to-year variation which is defined as a deviation from the climatological annual cycle of a meteorological quantity. It can be caused by a variation of an external forcing of the atmospheric circulation system, or can be generated internally within the system. On the other hand, intraseasonal variation is a low-frequency variation within a season, and it is basically considered to be a result of internal processes which may exist even under constant external conditions.

In this article, some observational facts on the intraseasonal and interannual variations of the polar stratosphere are presented, and the use of a hierarchy of numerical models to understand the stratospheric variations is reviewed systematically. Numerical models can be roughly divided into three classes based on their complexity; simple low-order models, medium mechanistic circulation models, and complex general circulation models. In order to understand the stratospheric variations, a hierarchy of stratosphere-only models have been used under an assumption of "slave stratospheric-variations" or "independent stratospheric-variations". Parameter sweep experiment, in which many trials of computations in parameter space are done by sweeping the value of a control parameter, is a powerful method to understand complex behavior in the models.

Recently, however, the importance of the coupled variability of the troposphere and the stratosphere was pointed out for the intraseasonal and interannual variations. In addition to numerical studies with simple or complex models, we have done some parameter sweep experiments with three-dimensional mechanistic circulation models to understand the troposphere-stratosphere coupled variability. All the effects of external forcings that might cause interannual variations can be excluded in the numerical experiments to focus only on internally generated variations within the coupled system. The obtained intraseasonal and interannual variations have some similar characteristics of the real atmosphere in some realistic parameter ranges. Roles of the interannual variations of the external forcings are discussed, which might be significant even if the amplitude is small.
\end{abstract}

\section{Introduction}

Time variations of the atmospheric state have two periodic components known as a diurnal cycle and an annual cycle, which are responses to the periodic variations of the external forcings due to the earth's rotation and

Corresponding author: Shigeo Yoden, Department of Geophysics, Kyoto University, Kyoto, 606-8502, Japan.

E-mail: yoden@kugi.kyoto-u.ac.jp

(C) 2002, Meteorological Society of Japan revolution about the sun, respectively. Examples of the former are land and sea breeze, mountain and valley winds, and thermal tides, which are periodic responses of the atmosphere to the diurnal differential heating by the sun on local or global scales. A monsoon climate with dry and rainy seasons is a clear example of the annual cycle.

Intraseasonal and interannual variations are defined as deviations from the periodic annual response. Generally intraseasonal variation means low-frequency variation with week- 
to-week or month-to-month time scales, while interannual variation means year-to-year variation. Some part of these variations is a response to the time variations of the external forcings or boundary conditions of the atmospheric circulation system, while the rest is generated internally within the system.

The atmosphere can be divided into four layers by the vertical thermal structure, and the lowest two are the troposphere and the stratosphere. The time variations of each of these layers have been studied rather independently, partly because the heat capacity is very different between the layers and the adjacent ocean and land. In recent years, however, interactions between the troposphere and the stratosphere in the intraseasonal and interannual time scales have drawn attention to a possible stratospheric role in climate (WMO 1998). In this review article, current understanding of the time variations of the troposphere-stratosphere (T-S) coupled system is summarized from a viewpoint of the external or internal causes. The use of hierarchy of numerical models to understand the time variations is reviewed, focusing on that for the internal intraseasonal and interannual variations.

\section{Intraseasonal and interannual variations}

Figure 1 shows an example of the time variations in the stratosphere. Daily temperature at $30 \mathrm{hPa}$ level is shown for the North Pole (a), the equator (b), and the South Pole (c) with gray lines from 1979 to 1997 . A climatological annual cycle averaged for the 19 years over each calendar day is also shown with a thick solid line, together with the annual cycle of the other pole after 6 months shift (dashed line). The annual cycle is very different between the North and South Poles. For example, the lowest temperature in winter is about $15 \mathrm{~K}$ higher at the North Pole, mainly due to dynamical effects. On the other hand, the highest temperature is about $10 \mathrm{~K}$ higher at the South Pole. Nearly half of the difference in summer can be explained by the elliptic revolution of the earth, while the rest is due to dynamical effects. The timing of the warming from winter to spring is also different between them. The daily temperature at the North Pole has large intraseasonal variations in winter and early spring, while large variations are seen in spring at the South Pole. The large variations at the North Pole are direct results of the occurrence of stratospheric sudden warming (SSW) events, which are associated with a rapid breakdown of the polar vortex from a strong, cold, and undisturbed state to another weak, warm, and disturbed one. See Randel and Newman (1998; Section 6.4) for details of the comparisons of day-to-day variability between the Northern Hemisphere $(\mathrm{NH})$ and the Southern Hemisphere (SH).

In the equatorial lower stratosphere, influence of the Quasi-Biennial Oscillation (QBO) of the zonal mean zonal flow is dominant in the original time series of temperature, but the annual component is also discernible in the climatology shown in Fig. 1 (b). The lowest temperature is observed in early February. The low temperature in northern winter can be understood as a result of adiabatic cooling due to the stronger upward motion associated with the wave-induced meridional circulation in the $\mathrm{NH}$ as firstly pointed out by Yulaeva et al. (1994).

The interannual variations of the polar temperatures are seen more easily in the monthly mean temperature data. The left and the center panels in Fig. 2 show annual variations of the histograms of the monthly mean temperature at the South Pole and at the North Pole with the same dataset as those used for Fig. 1. The interannual variation is very large in October and November at the South Pole, while it is largest in January, February and March at the North Pole. However, the frequency distributions are not very clear due to limitation on the data length. If we draw such histograms for the North Pole with a longer dataset over 45 years compiled at Free University Berlin (right panel), we can see some hints of skewed distribution for winter months. See Randel and Newman (1998; Section 6.5) again for details of the comparisons of interannual variability between the $\mathrm{NH}$ and the $\mathrm{SH}$.

Part of these interannual variations is a response to the time variations of external forcings or boundary conditions of the atmospheric circulation system as described in Labitzke and van Loon (1999), while the rest is generated internally within the system. Figure 3 illustrates important external forcings to the T-S coupled system. Although their relative importance is uncertain, well known natural forcings with 

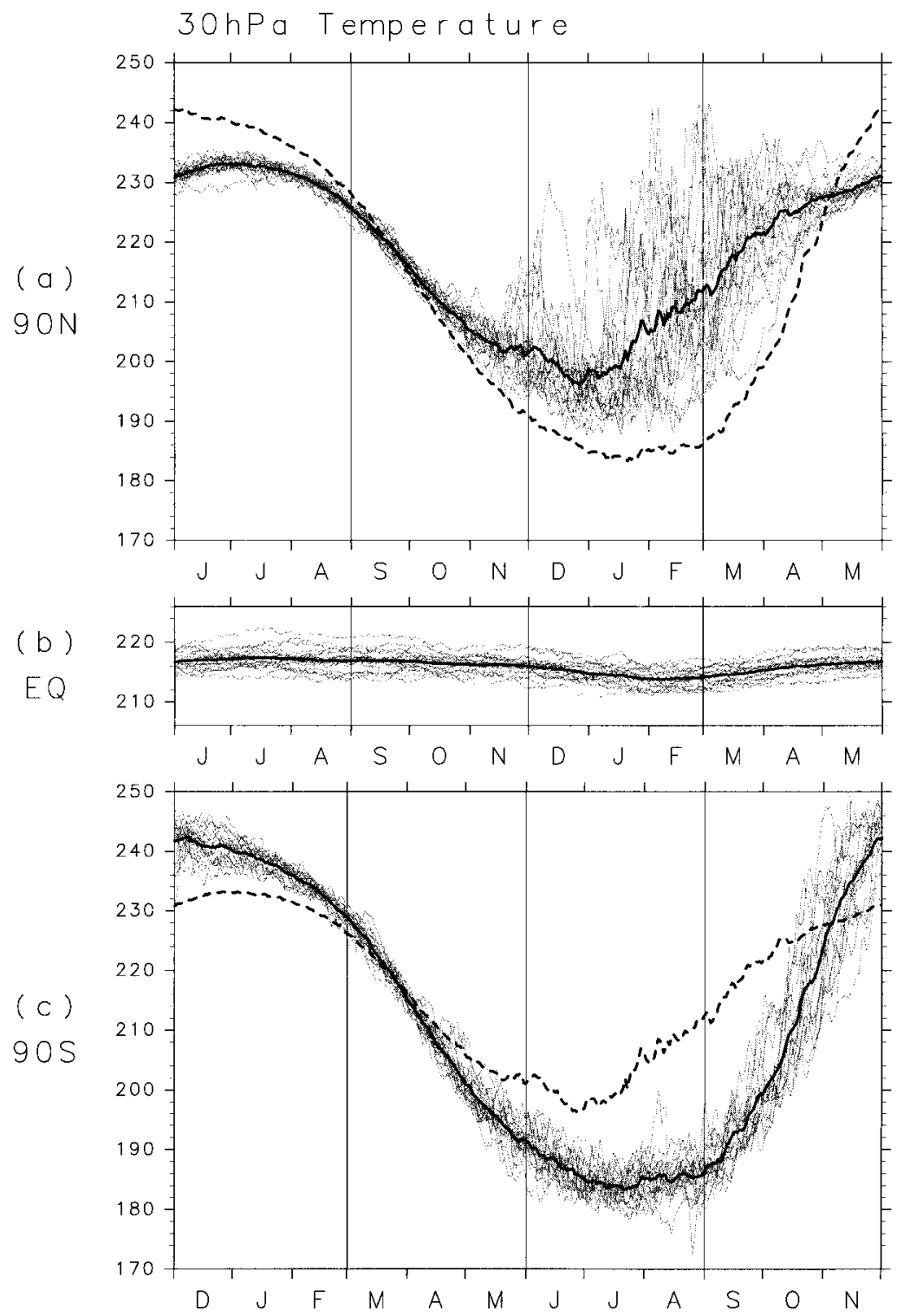

Fig. 1. Seasonal variation of daily temperature at $30 \mathrm{hPa}$ at the North Pole (a), the equator (b), and the South Pole (c) drawn with NCEP/NCAR reanalysis data for 1979-1997. The abscissa (time axis) for the South Pole (c) is shifted by 6 months to compare the seasonal variation directly. Thick solid line is the 19-year average for each calendar day, and dashed line is the same average for the other pole with 6 months shift.

interannual time scales are 11-year solar cycle and intermittent eruptions of volcanos; the former is very regular while the latter is irregular.

Sea-surface and land-surface conditions (e.g., temperature, roughness, snow/ice, biomass, ...) show significant interannual variations due to much larger thermal inertia of the ocean and land. These variations are fundamentally natural variations of the boundary conditions of the atmospheric system. However, they should be considered as internal variations of the coupled system of the atmosphere, ocean, land, and so on (see e.g., Trenberth 1992). For example, El Niño/Southern Oscillation is an inter- 


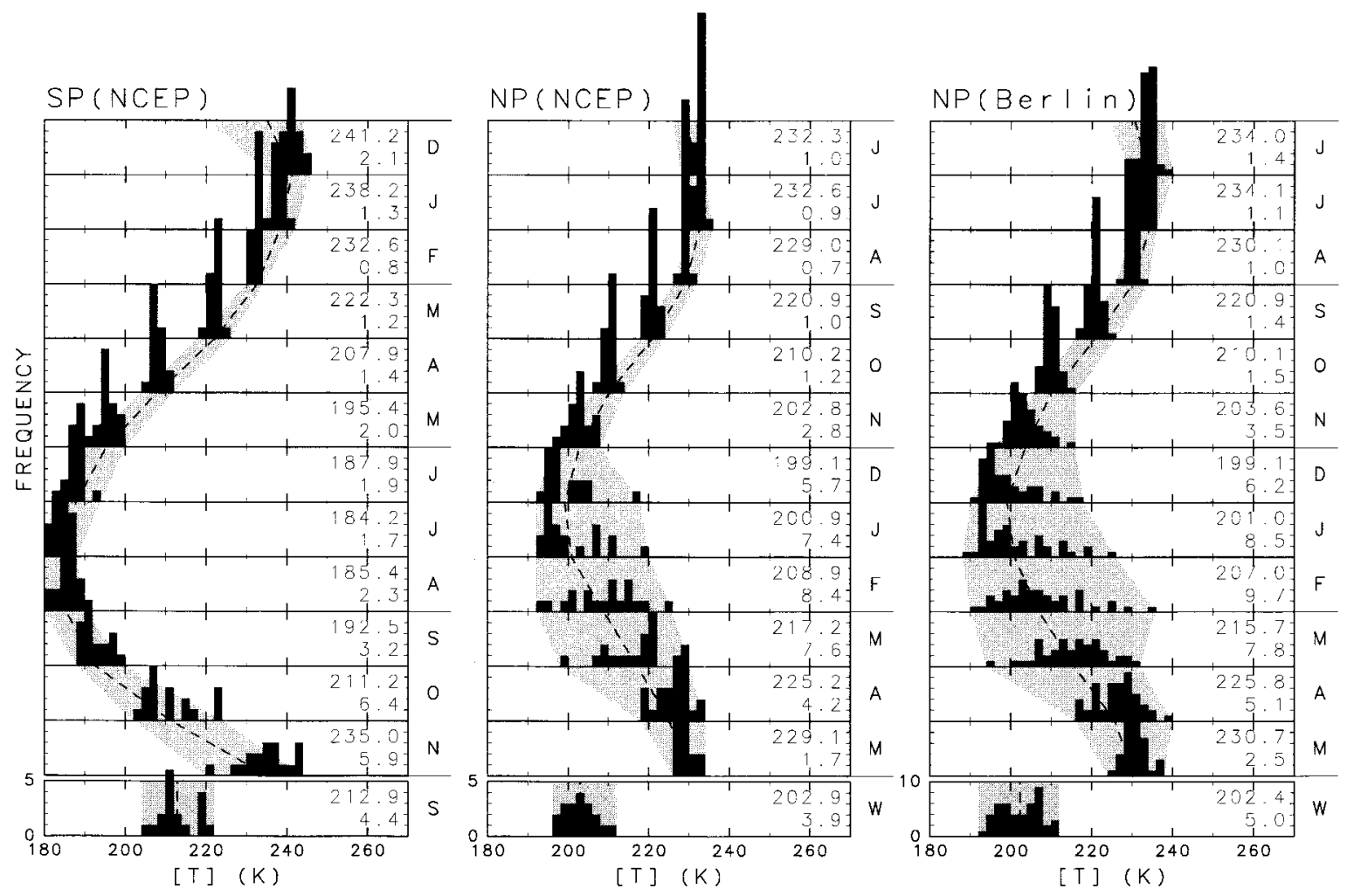

Fig. 2. Seasonal variation of histograms of the monthly mean temperature at $30 \mathrm{hPa}$ at the South Pole (left) and the North Pole (center) drawn with NCEP/NCAR reanalysis data for 1979-1997, and that for the North Pole with a longer dataset from July 1955 to October 2000 compiled at Free University Berlin (right; through the courtesy of Professor K. Labitzke). Dashed line denotes climatological annual cycle and shade shows the variable range. Two numbers for each month denote the climatological mean (top) and the standard deviation (bottom) of the monthly mean temperature. Frequency distributions for a seasonal mean are also displayed in the bottom: spring mean for the South Pole and winter mean for the North Pole.

nal variation of the atmosphere-ocean coupled system in the tropical Pacific (e.g., Philander 1990).

The QBO in the equatorial stratosphere is basically caused by the interaction between the zonal mean zonal flow and several kinds of waves, such as Kelvin, Rossby-gravity, inertiagravity, and smaller-scale gravity waves, propagating from the troposphere (e.g., Baldwin et al. 2001). The equatorial QBO could be considered as variation of a lateral boundary of the mid-latitude stratosphere, because interaction between the equatorial region and midlatitudes is primarily one way on that time scale, although the QBO is influenced by the mean meridional circulation driven by extratropical wave forces (Dunkerton 1991). Note that a QBO-like oscillation can be obtained even in a simplified general circulation model (GCM) without large-scale wave sources in midlatitudes (Horinouchi and Yoden 1998). The influence of the equatorial QBO on the winter stratospheric circulation was firstly pointed out by Holton and Tan $(1980,1982)$. Their composite analysis with a 16 -year data shows that the stratospheric polar vortex is weaker, warmer, and more disturbed during the easterly phase of the QBO. They argued the modification of the planetary wave propagation in the stratosphere by the equatorial QBO winds.

In addition to these natural forcings, there are some anthropogenic forcings such as the increases of greenhouse gases, aerosols, or chemical constituents related to the stratospheric 


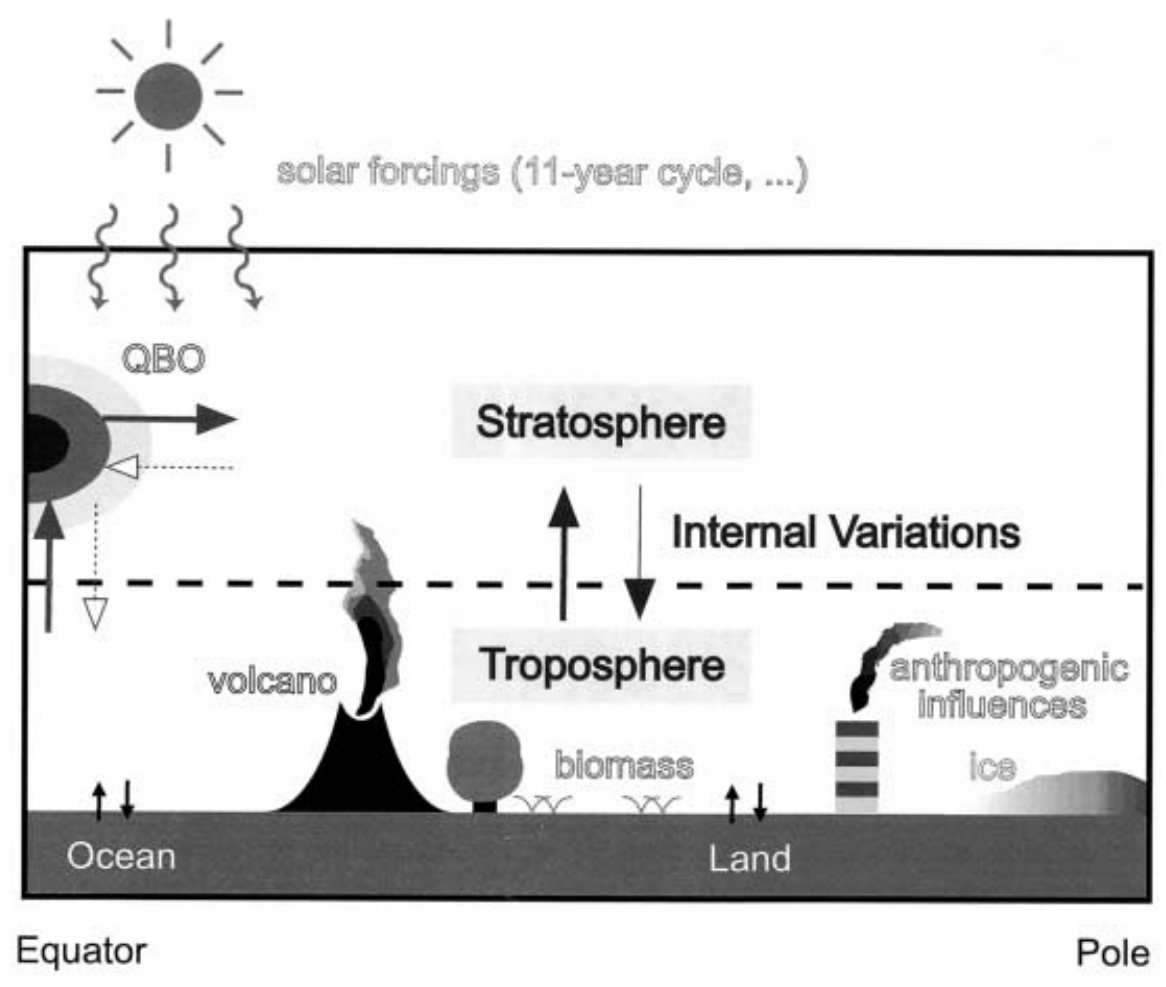

Fig. 3. External forcings to the troposphere-stratosphere coupled system. See text for details.

ozone decrease. Responses to these anthropogenic forcings are usually called "trends", because they increase monotonically in time. For example, stratospheric temperature has cooling trends of $-0.75 \mathrm{~K} /$ decade in the $20-35 \mathrm{~km}$ region and $-2.5 \mathrm{~K} /$ decade in the upper stratosphere (Ramaswamy et al. 2001). Both wellmixed greenhouse gases and ozone changes contribute in an important manner to the cooling. Variations of the biosphere, which may interact with the atmosphere in several ways, are mainly caused naturally, but anthropogenic effects such as deforestation may not be ignored.

On the other hand, there may exist internal interannual variations, because the atmosphere is a highly nonlinear system. For example, irregular non-periodic responses are unexceptionally obtained in multi-year integrations of a GCM with climatological boundary conditions which vary in time with a purely annual cycle (see e.g., Lau 1992). Generally, external forcings are not so significant in the intraseasonal time scales. Intraseasonal variation is basically considered to be a result of internal processes which may exist even under constant external conditions. Such variation may be a linear periodic oscillation or nonlinear variation (periodic, quasi-periodic, or chaotic) produced in the atmospheric circulation system.

The time variations discussed in this section can be classified systematically with some simple numerical models (Yoden 1997).

\section{Hierarchy of numerical models to understand the stratospheric variations}

Numerical models have been used widely in atmospheric research and forecasting in the past half century in accord with the development of computers (see e.g., Randall 2000). They can be roughly divided into three classes, "simple", "medium", and "complex" models, based on their complexity or degrees of freedom, which is the number of dependent variables after spatial discretization. The importance of balanced attack with a hierarchy of numerical models in meteorological research was pointed out by Hoskins (1983) a long time 


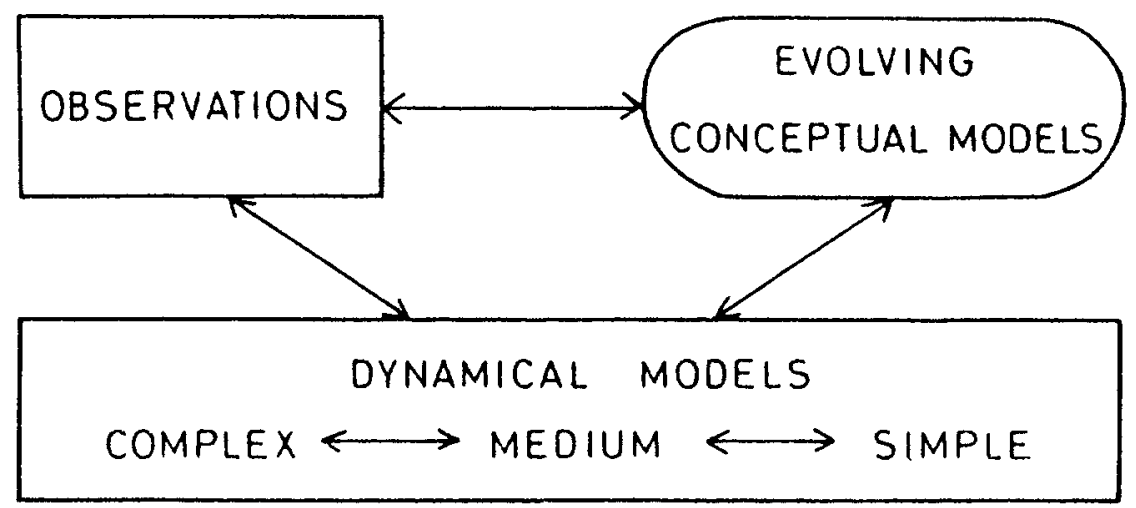

Fig. 4. A schematic illustration of the optimum situation for meteorological research (Hoskins 1983).

ago. Figure 4 illustrates the optimum situation he thought, in which all levels of modeling interact well with each other and with observations of the atmosphere to produce an evolving conceptual background, while this is used to aid in diagnosis of atmospheric and complex model behavior. In the past two decades, his advocacy has much influenced our numerical studies and those of others (see e.g., Andrews 2000; Section 8.1). We have carried out numerical experiments with a hierarchy of models, in order to understand intraseasonal and interannual variations in the polar stratosphere as shown in Figs. 1 and 2, and to investigate their dynamical linkage to the troposphere.

In the past, only simple low-order models (LOMs) with $\mathrm{O}\left(10^{0 \sim 1}\right)$ dependent variables could be used in "parameter sweep" experiments due to the limitation of computing resources. A pioneering work with an LOM was done by Lorenz (1963) to study the stepwise transition from steady axisymmetric flow to nonperiodic turbulent flow via vacillation in the rotating annulus experiments with radial differential heating (e.g., Hide and Mason 1975); he reduced a quasi-geostrophic two-layer system into 14-variable ordinary differential equations (ODEs), in order to carry out many trials of computations in a parameter space. Yoden (1987a,b, 1990) performed parameter sweep studies on the intraseasonal and interannual variability in the stratosphere with an LOM introduced by Holton and Mass (1976, hereafter referred to as HM). Some important results are summarized in the next section. We have to be careful of spurious results due to severe truncations in the spatial discretization, but LOMs are still useful for conceptual description or for illustration of the basic dynamics with limited components.

Nowadays it has become possible to use full dynamical models with $\mathrm{O}\left(10^{4 \sim 5}\right)$ variables for parameter sweep experiments. We call such models mechanistic circulation models (MCMs). Some idealization of physical processes in these models helps us to understand the essential dynamics. The resolution is sufficiently high that we do not need to worry about the truncation effect. Recently we used an MCM to investigate internal variations of the T-S coupled system (Taguchi et al. 2001; Taguchi and Yoden 2002a,b,c), and some highlights are shown in Section 6.

For quantitative arguments, more complex GCMs with $\mathrm{O}\left(10^{4 \sim 7}\right)$ variables are necessary, in which sophisticated physical parameterization schemes should be adopted. Some middle atmosphere GCMs with a high top boundary have been used to investigate the effects of the stratosphere on the tropospheric climate (Pawson et al. 2000). A future challenge is realistic simulation of the whole atmosphere, including the middle and upper atmosphere, as well as the troposphere, in order to be able to address a wide range of new problems dealing with couplings between widely separated layers of the atmosphere (Blackmon et al. 2001). Time integrations for hundreds of model-years are done in some studies with these full GCMs, but parameter sweep experiments or ensemble 


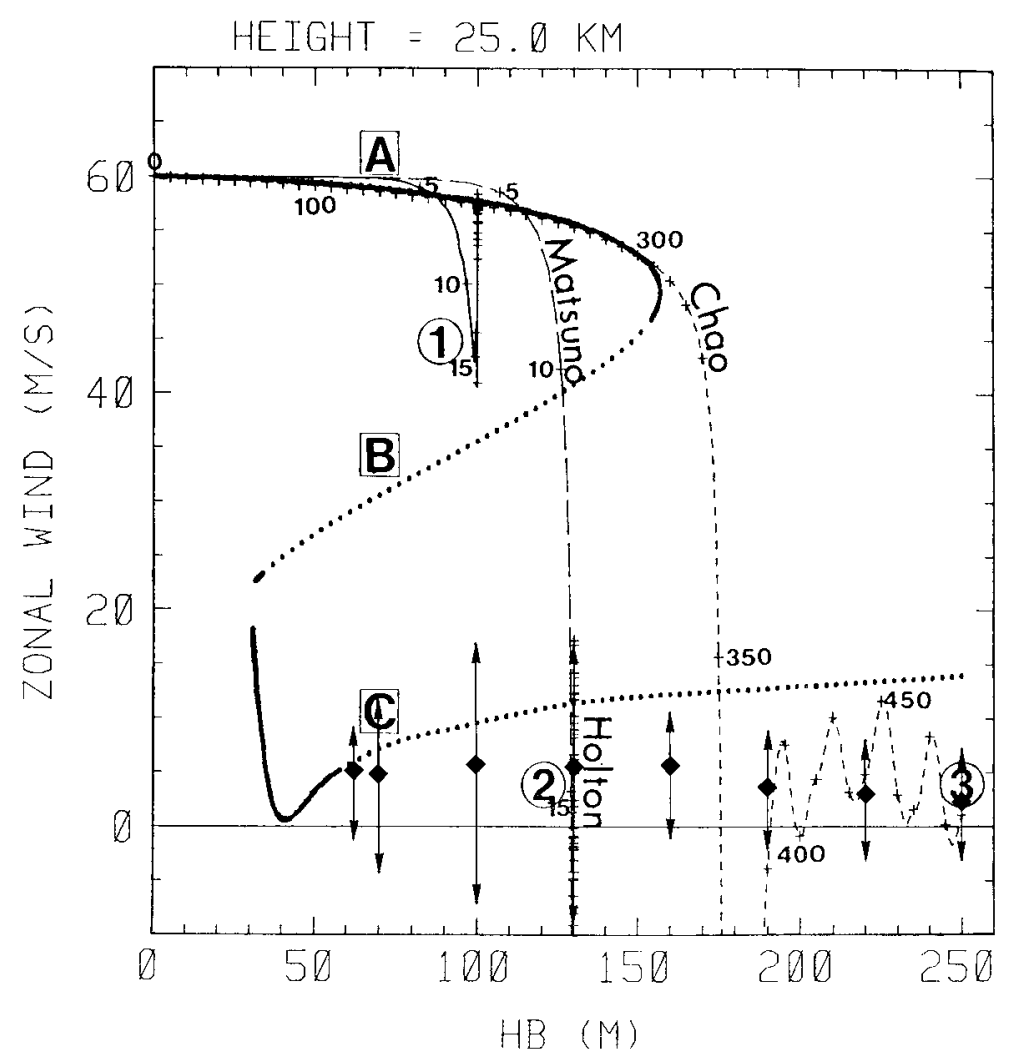

Fig. 5. Bifurcation diagram of the HM model and trajectories of three time-dependent solutions projected onto the same plane; the abscissa is the external parameter $h_{B}$ and the ordinate is a dependent variable of the model, the mean zonal wind at $z=25 \mathrm{~km}$. Steady solution branches (A)-(C) are denoted by solid lines for stable solutions and dotted lines for unstable ones. Variable range of stable vacillations (periodic solutions) are drawn with vertical arrows with two heads, and diamonds denote the time mean values. Three lines (1)-(3) are responses to different wave forcings at the bottom boundary. (1) $h_{B}=100 \mathrm{~m} \times[1-\exp (-t / T)]$ with $T=2.5 \times 10^{5} \mathrm{~s},(2) h_{B}=130 \mathrm{~m} \times$ $[1-\exp (-t / T)]$, (3) $h_{B}=0.5 \mathrm{~m} \mathrm{day}^{-1} \times t$. Symbol + is put every 5 days for (1) and (2) and 10 days for (3). Unit of label is written in days.

runs with large numbers of members are still limited, even in the most advanced computational environment.

\section{Use of stratosphere-only models}

Some simplified models of the interaction between the zonal mean zonal flow and planetary waves propagating from the troposphere have been developed to study the stratospheric variations including SSW events. The HM model is such an LOM which has been used by many authors. It is a highly-truncated spectral model of quasi-geostrophic flow in a mid-latitude $\beta$ channel, that is, a one-dimensional height-only model with zonal and a single-wave longitudi- nal resolution. After vertical discretization it becomes 81-variable system of ODEs. Important external parameters in the HM model are the intensity of the mean zonal flow forcing, $d U_{R} / d z$, and the wave amplitude at the bottom boundary placed near the tropopause level, $h_{B}$.

Yoden (1987a) investigated the bifurcation properties of the HM model with a sweep parameter, $h_{B}$. Figure 5 is a bifurcation diagram which shows the existence of multiple stable solutions for a given value of $h_{B}$. As the ordinate is the zonal mean zonal wind at $z=$ $25 \mathrm{~km}$, or a measure of the intensity of polar vortex, the solution branch (A) corresponds to a strong and cold polar vortex, the branch $(\mathrm{C})$ 
does to another weak and warm polar vortex, and the branch (B) intermediate one. Linear stability analysis shows two solutions of (A) and (C) are stable for a finite range of $h_{B}$. Stable periodic solutions, a series of stratospheric vacillations originally found by $\mathrm{HM}$, also branch off from the steady solution (C) at $h_{B} \sim 59 \mathrm{~m}$. The stable steady solution (A) and the stable periodic solution coexist, depending on the initial condition, in the parameter range of $59 \mathrm{~m}<h_{B}<157 \mathrm{~m}$.

Such concept of multiple stable solutions can be applied for understanding the intraseasonal variability of the $\mathrm{NH}$ winter stratosphere. Yoden (1987a) reinterpreted some theoretical models of SSW with the same framework of the HM model. The essence of Matsuno's (1971) theory is impulsive initiation of a wave forcing in the troposphere; transient response to the increase of $h_{B}$ for a short time interval may cause a rapid transition from the state of strong and cold polar vortex to the other weak and warm state as denoted by a broken line with a label "Matsuno". Another theory of SSW is the stratospheric vacillation found by HM (denoted by "Holton"). HM showed that a periodic variation of the stratosphere which mimics repeated occurrence of SSW events with a period of 50100 days may exist even for a time-constant $h_{B}$. The vacillation is a nonlinear internal variation of the mid-latitude stratospheric system with fixed external conditions.

These two theories made very opposite assumption on the dynamical linkage between the troposphere and the stratosphere. Matsuno (1971) assumed "slave stratosphericvariations"; the stratospheric variations are caused by the variation of its bottom boundary, that is, the troposphere, without any stratospheric influence on the troposphere. On the other hand, HM assumed "independent stratospheric-variations"; the stratospheric variations are possible for a time-constant bottom boundary condition. The catastrophe SSW theory by Chao (1985), which assumes very gradual increase of $h_{B}$ exceeding a critical value, belongs to the group of slave stratospheric-variations. Plumb's (1981) instability theory of the distorted polar vortex is another SSW theory in the framework of independent stratospheric-variations, although his theory cannot be directly applied to the HM model be- cause of its different bottom boundary condition.

In addition to the steady and periodic solutions, quasiperiodic and chaotic (nonperiodic) solutions are found in the same HM model by Christiansen (2000a) with a thorough parameter sweep experiment. Scott and Haynes (2000) investigated internal vacillations in a stratosphere-only model with an intermediate size of $\mathrm{O}\left(10^{3}\right)$, which is a two-dimensional latitude-height primitive equation model with zonal and wave-1 longitudinal resolution. They found steady and vacillating flow regimes with constant external conditions and multiple flow equilibria as obtained in the HM model, and they pointed out the importance of the meridional structure of the zonal mean zonal flow in determining fluxes of wave activity and consequently in determining the mean flow evolution. Scaife and James (2000) used a threedimensional, global primitive-equation model of the stratosphere and mesosphere to investigate such internal variations by a model with isotropic horizontal resolution. The fully nonlinear MCM exhibits three flow regimes under fixed perpetual-January conditions, depending on the amplitude of wave forcing at the bottom boundary near the tropopause; strong steady polar vortex for small wave-amplitude forcing, strong but unsteady polar vortex for moderate wave-amplitude forcing, and vacillations (or, repeated occurrence of SSW events with a time-interval of 30-50 days) for large waveamplitude forcing. Christiansen (1999) also obtained stratospheric vacillations in GCM experiments with hypothetical time-independent tropospheres.

Based on these studies with a hierarchy of numerical models, we can conclude with confidence that independent stratosphericvariations may exist for the bottom boundary condition of a time-constant wave forcing near the tropopause. Such a bottom boundary condition may not be very realistic, but these studies under an idealized dynamical situation are useful to understand the basic nature of the stratospheric variations.

Seasonal and interannual variations of the stratospheric circulation have been studied theoretically with some independent stratosphericvariation models. Holton and Wherbein (1980, 1981) investigated the annual cycle of the zonal 

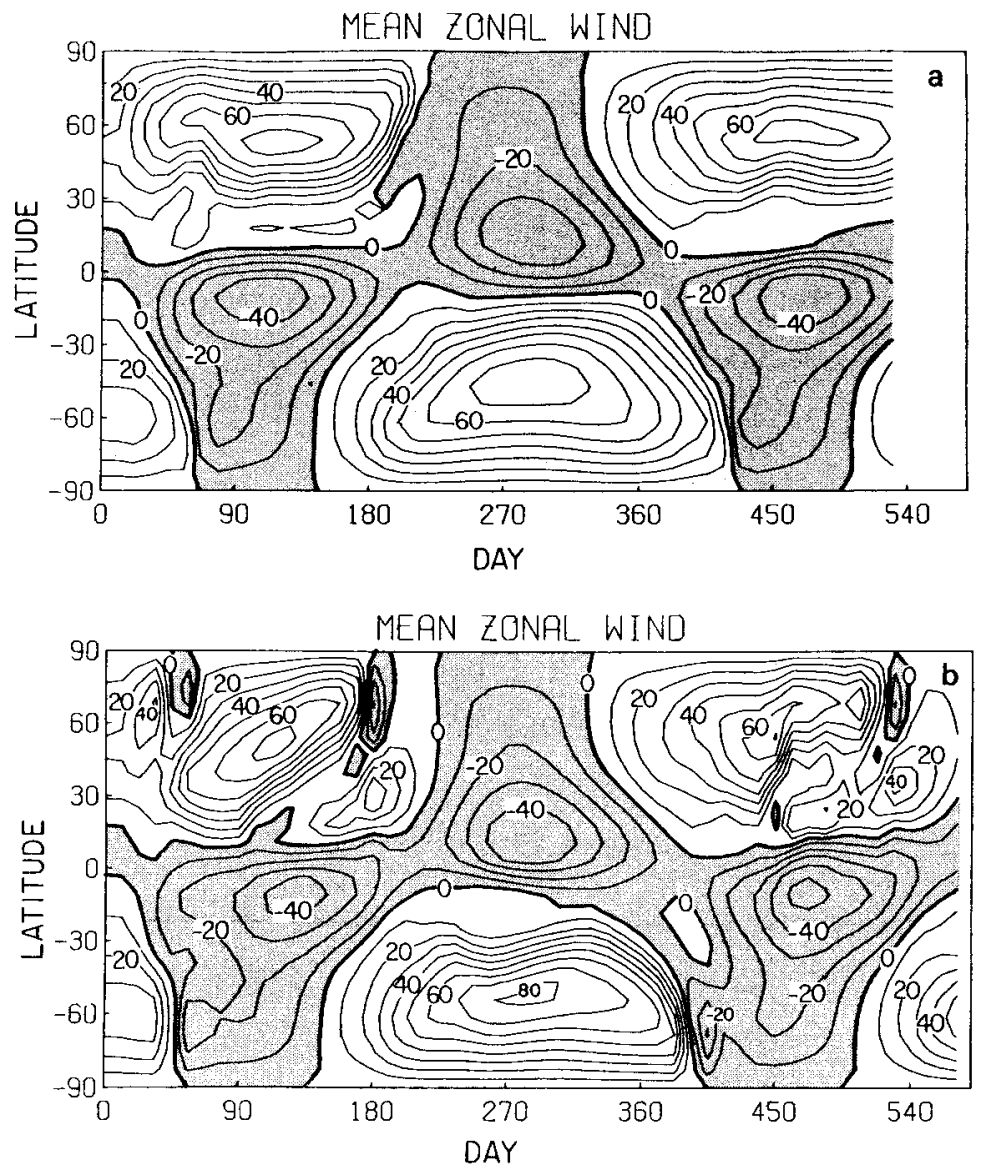

Fig. 6. Annual cycle of the zonal mean circulation in the middle atmosphere (Holton and Wherbein 1981). Time-latitude sections of the mean zonal wind $[\mathrm{m} / \mathrm{s}]$ at $51 \mathrm{~km}$ level for (a) subcritical wave forcing and (b) supercritical wave forcing. Easterlies are shaded. A 360-day year is used for simplicity, and Day 0 corresponds to the Northern Hemisphere autumn equinox.

mean circulation of the middle atmosphere with a severely truncated global primitive-equation model, which is similar to that lately used by Scott and Haynes (1998, 2000, 2002). Figure 6 shows annual cycles of the zonal mean zonal wind near the stratopause for (a) subcritical wave forcing at the bottom boundary and (b) supercritical wave forcing. In the supercritical case, the flow undergoes substantial transient intraseasonal variations, including both major and minor SSW events.

Much simple height-only $\beta$-channel models have also been used to understand the nature of seasonal and interannual variations (e.g., Wakata and Uryu 1987; Plumb 1989; Yoden 1990). Yoden (1990) performed an experiment by varying $d U_{R} / d z$ in the HM model periodi- cally with an annual component for various values of $h_{B}$, but obtained only periodic annual response, or seasonal variation. No example of interannual variations (i.e., deviations from the seasonal variation) was obtained. The seasonal variation is qualitatively different depending on the magnitude of $h_{B}$, and the difference resembles that of the climatological seasonal march between the $\mathrm{NH}$ and the $\mathrm{SH}$; intraseasonal variations of the zonal mean quantities and wave activity are large in winter in large $h_{B}$ cases, while those are large in equinox seasons in small $h_{B}$ cases. Qualitatively similar results which depend on the amplitude of wave forcing are also obtained by Scott and Haynes (2002) with a primitive-equation model with both height and latitude structure. They found 


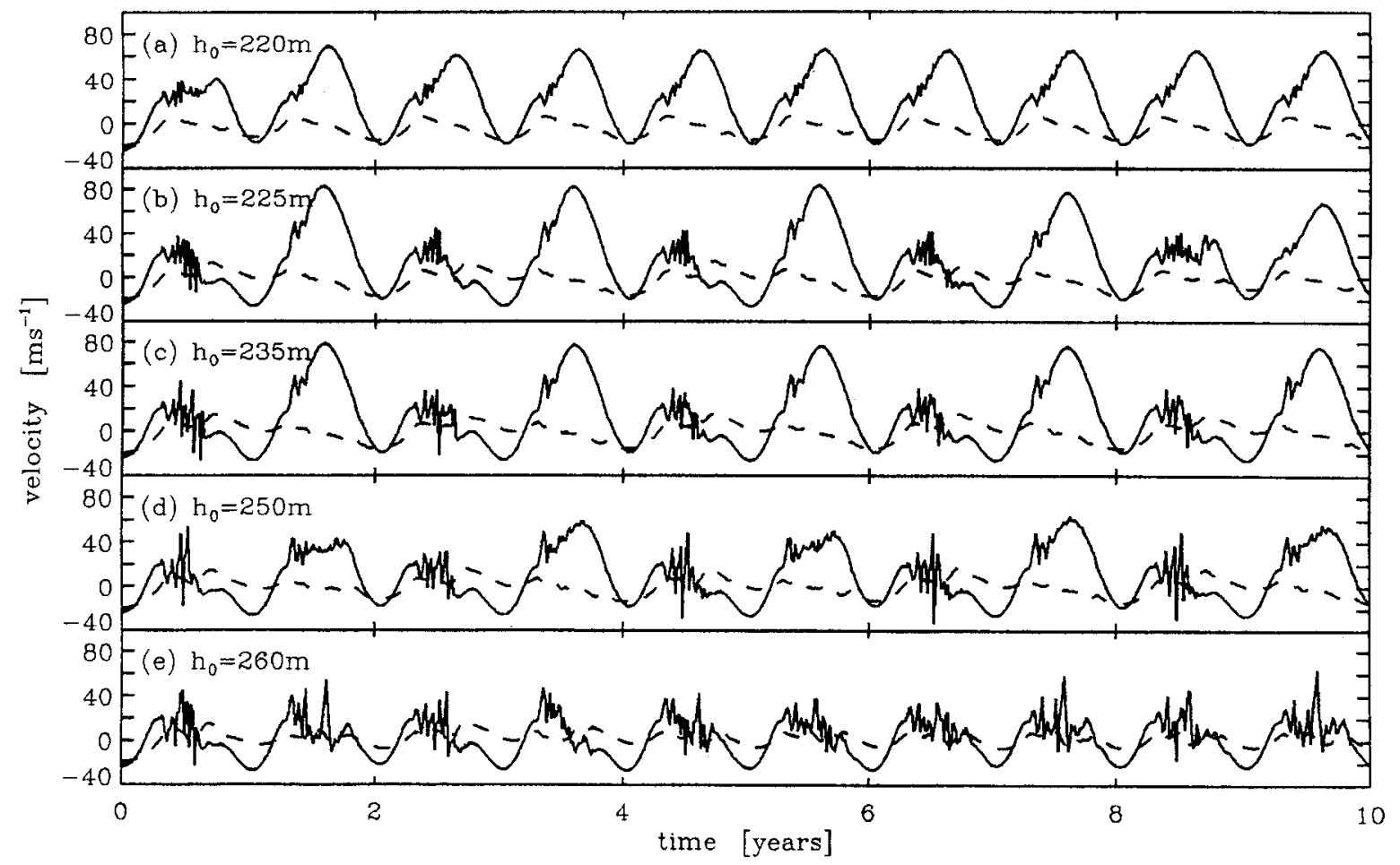

Fig. 7. Evolution of the mean zonal wind, at the latitude of $\phi=60^{\circ}$ and the height of $z=40 \mathrm{~km}$ (solid curves) and at $\phi=20^{\circ}, z=25 \mathrm{~km}$ (dashed curves), for ten year integrations with five different wave forcing amplitudes $h_{0}$ (Scott and Haynes 1998). Time $t=0$ corresponds to midsummer.

that two possible winter and late winter evolutions exist for the same forcing amplitude more typical of the NH. Which of these is selected depends on details of the forcing in early winter.

By using a similar primitive-equation model, Scott and Haynes (1998) found interannual variations even for a periodic annual forcing. Figure 7 shows the time evolution of the mean zonal wind in the mid-latitude stratosphere for ten year runs with various values of the wave forcing amplitude, $h_{0}$. For $h_{0}=220 \mathrm{~m}$ the response is annually periodic, with strong westerly in winter. As $h_{0}$ is increased, there is a transition to a biennial response characterized by alternate appearance of dynamically quiescent strong polar vortex and dynamically disturbed weak one in winter season. For larger $h_{0}$ there appears a more gradual transition from biennial response to random interannual variability. The internal interannual variability arises owing to the longer "memory" of the stratospheric flow at low latitudes (dashed curves). A given wind signal at low latitudes is less affected by radiative damping due to the smaller Coriolis parameter. In other words, relaxation time for zonal angular momentum is much longer than that for temperature in the low latitudes. Internal variability due to the longer memory of low latitude winds might have a role in the interannual variability in the real stratosphere.

As for highly-truncated $\beta$-channel models, Christiansen (2000a) recently showed by a thorough parameter sweep experiment that internal interannual variations do exist in the same model framework of Yoden (1990). Chen et al. (2001) also demonstrated that such interannual variations can be obtained if antisymmetric mode about the channel center is included into the model with a different value of relaxation time.

Some stratosphere-only models have been used to investigate the influence of the equato- 
rial QBO on the winter stratospheric circulation (e.g., Holton and Austin 1991; O'Sullivan and Young 1992; O'Sullivan and Dunkerton 1994; Gray et al. 2001; Gray et al. 2002). For example, Holton and Austin (1991) performed parameter sweep in a Matsuno-type SSW (or, slave stratospheric variation) experiment by changing the amplitude of planetary wave forcing at the $100 \mathrm{hPa}$ level with mean zonal flow conditions corresponding to the westerly and easterly phases of the QBO, respectively. The phase of the QBO had little effect on the results in weak wave cases and strong wave cases. In intermediate wave cases, on the other hand, the SSWs occurred earlier and wave variability was stronger for the easterly phase, in qualitative agreement with the observations by Holton and Tan (1980, 1982). The other works except for Gray et al. (2002) assumed similar experimental framework of slave stratospheric variations by specifying time-dependent planetary wave forcing. These numerical experiments confirmed the Holton-Tan relationship, by showing the sensitivity of the wintertime polar circulation to the phase of the QBO and the modification of the planetary wave propagation by the QBO. On the other hand, Gray et al. (2002) assumed a time-constant planetary wave forcing. They showed that there is no strong tropospheric control of the stratospheric response in intermediate wave cases, in which the equatorial QBO winds and the "initial conditions" in autumn and early winter are important to determine the seasonal development of the polar vortex.

\section{Troposphere-stratosphere coupled variation}

Planetary waves and gravity waves generated in the troposphere propagate upward carrying zonal angular momentum. Breaking of these waves and other dissipative eddy effects produce mean zonal forces in the stratosphere and mesosphere to cause surprising phenomena such as SSWs and QBO (see e.g., Shepherd 2000). These are typical examples of upward influence from the troposphere to the stratosphere. Time variations of these waves near the tropopause are prescribed in slave stratospheric-variation models, while timeconstant waves are assumed in independent stratospheric-variation models. However, any process of downward influence from the stratosphere to the troposphere can not be incorporated into these stratosphere-only models.

Recently, possible climate impacts of changes in the temperature, circulation, and minor chemical constituents of the stratosphere have been pointed out (e.g., WMO 1998; Blackmon et al. 2001). Thompson and Wallace (1998) showed that the leading empirical orthogonal function (EOF) of the wintertime sea-level pressure variability in the $\mathrm{NH}$, the Arctic Oscillation (AO), is the surface signature of modulations in the strength of the polar vortex in the troposphere and lower stratosphere. It is a deep signature of zonally-symmetric seesaw patterns of geopotential height, alternating between the polar region and mid-latitudes, with intraseasonal and interannual time scales. Such annular variability of the polar vortex is observed in all seasons in both hemispheres and the T-S coupling is stronger in the dynamically active season, namely winter in the $\mathrm{NH}$ or spring in the $\mathrm{SH}$ (Thompson and Wallace 2000). Downward propagation of the AO signature from the stratosphere to the troposphere was noticed in association with SSW events (Baldwin and Dunkerton 1999; Kuroda and Kodera 1999). Such stratospheric harbinger may be used as a predictor of tropospheric weather regimes (Baldwin and Dunkerton 2001). These studies are indicative of the importance of two-way interactions between the troposphere and the stratosphere.

Hartmann et al. (2000) summarized dynamical mechanisms by which the stratosphere can influence the troposphere; mass redistribution in the stratosphere, wave propagation, and potential vorticity induction.

The eddy induced-mean zonal forces given in the extratropical stratosphere produce a diabatic circulation which penetrates into the troposphere due to "downward control principle" described by Haynes et al. (1991). The downward influence is important in stratospheretroposphere exchange of mass and chemical species (Holton et al. 1995). Baldwin and Dunkerton $(1999,2001)$ discussed a possible mechanism of the mass redistribution in the upper troposphere or lower stratosphere, which controls the pressure below. An anomalous westward mean zonal force in middle to high latitudes induces poleward mean meridional 
circulation as stated above, with open streamlines at the surface (Haynes and Shepherd 1989). The low-level return flow therefore does not entirely cancel the transport of mass in the upper branch, and, as a result, surface pressure in the polar cap increases. This linear mechanism may be applied, in reverse, for an eastward body force anomaly to obtain pressure decrease in the polar cap.

Tropospheric circulation changes associated with SSW events have been reported since 1960's as case studies; a recent study by Kodera and Chiba (1995) showed changes in the direction of the meridional propagation of planetary waves in the troposphere as well as the intensification of the upward propagation in the stratosphere. Kuroda and Kodera (1999, 2001) investigated longer time-scale intraseasonal variations in cold season in the both hemispheres and found poleward and downward propagation of mean zonal wind anomalies from the subtropical stratopause to the highlatitude troposphere (Polar night Jet Oscillation; PJO) through interaction with planetary waves. The downward propagation of wind anomalies into the troposphere may influence the wave activity trapped in the troposphere, because the propagation of planetary waves between the troposphere and stratosphere is very sensitive to the vertical shear of the mean zonal wind near the tropopause (Chen and Robinson 1992). It was also pointed out that the propagation route of planetary waves is very sensitive to the AO-related variations (Hartmann and Lo 1998; Limpasuvan and Hartmann 1999, 2000; Kodera and Kuroda 2000a).

If we use the invertibility principle for potential vorticity with some kinds of balance condition, reference state, and boundary conditions (Hoskins et al. 1985), we can diagnose the flow field associated with a given potential vorticity distribution. Hartley et al. (1998) performed so-called piecewise potential vorticity inversions to argue that redistribution of potential vorticity in the lower stratosphere induces perturbations in key meteorological fields in the upper troposphere. Black (2002) extended the work to show that during the AO surface climate variations are directly forced by changes in the strength of the stratospheric polar vortex.

\section{Use of troposphere-stratosphere coupled models}

Boville (1984) performed a GCM experiment on the downward influence from the stratosphere to the troposphere. He compared a control simulation of a T-S GCM with a degraded simulation in which one of the physical parameterizations was altered in the stratosphere to give a poorer simulation of the polar night jet. Statistically significant differences in the tropospheric simulation were found not only in the structures of stationary planetary waves but also in the activity of transient synoptic eddies.

Since his pioneering work many numerical studies with troposphere-stratosphere (-mesosphere) GCMs have been done to investigate the two-way interactions between the troposphere and stratosphere. Internal variability of the T-S coupled system has been investigated by performing long time integrations with constant boundary and external conditions (e.g., Pawson et al. 1995; Yoden et al. 1996; Yoden et al. 1999). Yoden et al. (1999) performed a composite analysis of 64 SSW events obtained in a perpetual January integration for 7200 days. Figure 8 is the composite time-height sections of normalized zonal-mean temperature variations in high latitudes for zonal wave- 1 events (a) and for zonal wave-2 events (b). In both groups the preconditioned states in the upper troposphere and lower stratosphere are similar before SSW events; the zonal mean temperature is lower than normal in the polar region, and the zonal mean zonal wind is stronger than normal in the middle and high latitudes. A different dynamical behavior between the two groups is found during and after SSW events; for zonal wave-1 events the preconditioned states continue even after the events near the tropopause, while signals of SSW descend to the upper troposphere after the zonal wave-2 events. Note that such a large number of SSW events can be obtained only by a long time integration of numerical models. As for the study of intraseasonal variations related to the $\mathrm{AO}$ in the T-S coupled system, Yamazaki and Shinya (1999) and Christiansen (2000b, 2001) used GCMs under perpetual winter conditions or climatological seasonal variations, and showed that the $\mathrm{AO}$ is an atmospheric internal mode. Yamazaki and 

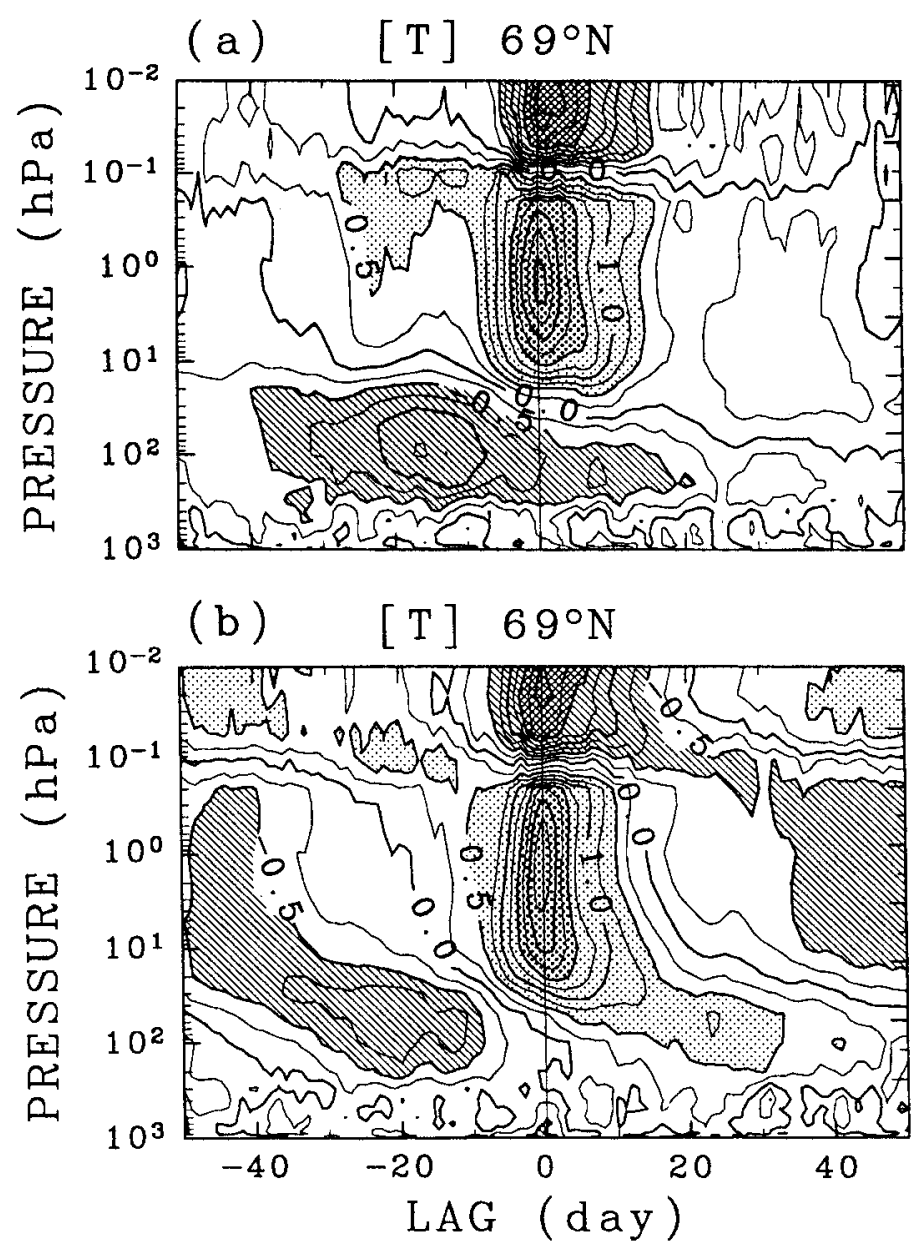

Fig. 8. Composite time-height sections of normalized zonal-mean temperature variations at $69^{\circ} \mathrm{N}$ for 34 zonal wave-1 SSW events (a) and for 30 zonal wave-2 events (b), taken from Yoden et al. (1999). In a 7200-day dataset obtained from a perpetual January integration of the Berlin GCM, 64 SSW events are defined based on the polar temperature at $p=1 \mathrm{hPa}$, and divided into the above two groups according to the relative strength of planetary waves of zonal wavenumber 1 and 2 in the stratosphere. A key day for each event is also defined as the day of the maximum, and the temperature anomaly normalized with its standard deviation at each level is composited for the key day ( $\operatorname{Lag}=0$ day). Contour interval is 0.25 . Normalized values greater than 0.5 are lightly shaded with dots and those greater than 1.5 are heavily shaded, while those less than -0.5 are lightly shaded with diagonal lines and those less than -1.5 are heavily shaded with cross lines.

Shinya (1999) also detected slowly propagating PJO with a period of $4-6$ months in a perpetual February run.

GCMs have also been used to investigate the responses of the T-S coupled system to the time variations of external forcings or boundary conditions of the system. Niwano and Takahashi (1998) investigated the influence of the equatorial QBO that was simulated in their GCM on the $\mathrm{NH}$ winter circulation, while Hamilton
(1998) investigated the effects of an imposed QBO using the GFDL SKIHI GCM. Both studies confirmed the Holton-Tan relationship and showed modest effects of the QBO to the winter troposphere. By using a coupled oceanatmosphere GCM, Kitoh et al. (1996) investigated interannual variability in the T-S circulations and its relationship with the variation of sea surface temperature (SST). Their model results show that interannual variations 
of the stratospheric polar vortex are related with the North Pacific SST through tropospheric circulation changes. Lahoz (2000) investigated the northern winter stratosphere variability simulated in a T-S version of The Met. Office Unified Model with observed SSTs from 1979 to 1998, and showed that the Unified Model reproduces the observed temperature trend with a fixed ozone climatology and fixed greenhouse gases. SSTs appear to influence the northern winter stratosphere mainly through changes in the atmospheric circulation. On the other hand, Shindell et al. (1999a) simulated recent northern winter climate trends, which is characterized by a systematic bias in one phase of the AO (Thompson and Wallace 1998), by assuming realistic increases in greenhousegas forcing. Shindell et al. (1999b) performed another GCM experiment to investigate the effect of 11-year solar cycle irradiance changes on climate, and demonstrated the importance of dynamical T-S coupling. In the model, circulation changes initially induced in the stratosphere by the changes in solar irradiance and ozone subsequently penetrate into the troposphere. Hartmann et al. (2000) argued the possibility that stratospheric ozone depletion and greenhouse warming may interact to produce rapid climate change through internal variations related to the AO.

Kodera and Kuroda (2000b) developed an LOM of T-S coupled system, i.e., the other end of a hierarchy of T-S models, to understand the mechanism of the coupled variability; they extended the HM model by including an antisymmetric meridional mode as Chen et al. (2001) to incorporate the subtropical jet. Vacillations with some realistic periods are obtained for moderate wave forcing amplitudes given at the surface. Zonal wind anomalies generated in the subtropical stratopause propagate downward into the polar troposphere, which provoke changes in the meridional propagation of tropospheric planetary waves. However, such simplified models do not include the effects of baroclinic disturbances in the troposphere, which is important to generate the planetary waves, in addition to topographic and thermal forcings (e.g., Held 1983; Andrews et al. 1987).

Recent progress in computing facilities has enabled us to perform some parameter sweep experiments, similar to those done with LOMs over a decade ago, with three-dimensional MCMs in order to understand the T-S coupled variability. Taguchi et al. (2001) modified an atmospheric GCM by making some simplifications of physical processes; all the moist processes were taken out, the radiation code was replaced by a simple Newtonian heating/ cooling scheme, Rayleigh friction was used at the surface, and sinusoidal surface topography was assumed in longitudinal direction with a single zonal wavenumber $m=1$ or 2 component only in the $\mathrm{NH}$. The model is a spectral primitive-equation model with 42 vertical levels from the surface to the mesopause, and its horizontal resolution is given by a triangular truncation at total wavenumber 21 in spherical harmonics. Thus the model has $\mathrm{O}\left(10^{5}\right)$ degrees of freedom and can describe baroclinic disturbances explicitly. All the effects of external forcings illustrated in Fig. 3 are excluded in our experiments, and relative importance of the planetary waves forced by the idealized topography is investigated thoroughly by making a parameter sweep on the topographic amplitude $h_{0}$. For all the range of $h_{0}$, the amplitude and latitudinal structure of the forced wave vary in time irregularly in the troposphere by interactions with the mean zonal flow and baroclinic disturbances (Taguchi et al. 2001). Thus, such irregular behavior of forced planetary waves is not well incorporated into stratosphere-only models, neither slave stratospheric-variation models nor independent stratospheric-variation ones.

Under a purely periodic external condition of the annual thermal forcing, 10 runs of 100-year integrations were done with different topographic amplitude of $0 \mathrm{~m} \leq h_{0} \leq 3000 \mathrm{~m}$ (Taguchi and Yoden 2002a). Figure 9 shows the dependence of temperature variations in the polar stratosphere on $h_{0}$. When it is equal to zero or a small value, the interannual variation is very small, particularly in winter. The interannual variation becomes large in spring for $h_{0}=400 \sim 600 \mathrm{~m}$, which resembles the variation in the SH as shown in Fig. 1(c). If $h_{0}$ is increased further, the variation becomes large in winter. The results for $h_{0}=700$ or $1000 \mathrm{~m}$ resemble the variation in the NH. For small and intermediate forcing, SSW event does not take place in midwinter owing to the suppression of 

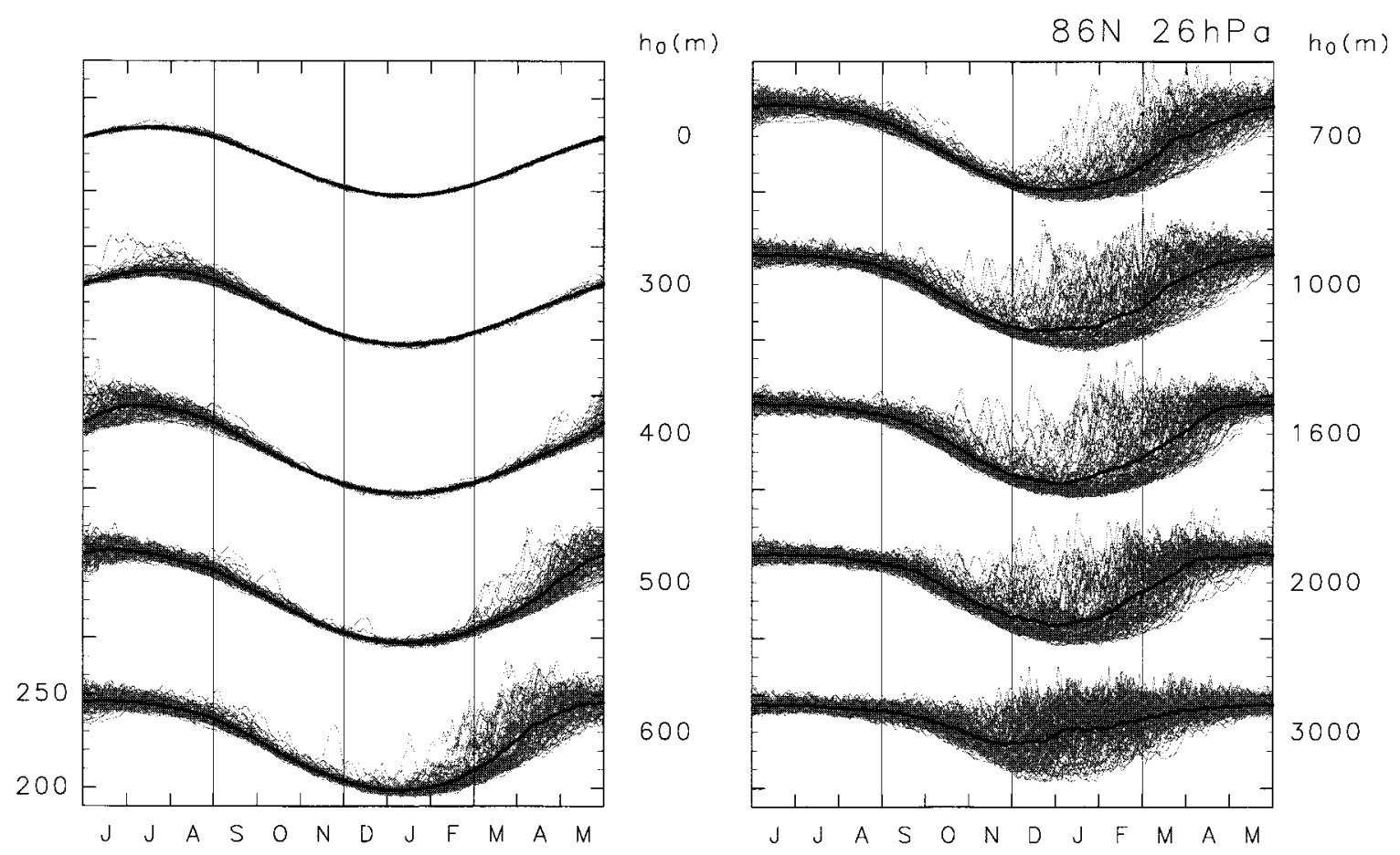

Fig. 9. Seasonal variation of daily temperature at $86^{\circ} \mathrm{N}$ and $26 \mathrm{hPa}$ for 10 runs of 100 -year integrations under a purely periodic annual forcing. The topographic amplitude $h_{0}$ of zonal wavenumber 1 is changed from $0 \mathrm{~m}$ to $3000 \mathrm{~m}$ as the experimental parameter. Thick line is the 100-year average for each calendar day.

wave activity in the stratosphere, in consistent with the previous studies with simple LOMs (Plumb 1989; Yoden 1990; Scott and Haynes 2002). Note the interannual variation in this experiment is caused only by the internal nonlinear processes in the T-S coupled system. It is a manifestation of the randomness of the phase of intraseasonal variations, or timing of the occurrence of SSW event, in each year, which randomness is caused by the irregular behavior of forced waves in the troposphere.

Taguchi and Yoden (2002b) also made 2 runs of 1000-year integrations under the same purely periodic annual forcing, which were performed by ensemble technique; 100-year integrations were done after one-year spin-up for each of 10 different initial states. In the millennium integrations, statistically reliable frequency distributions are obtained in the monthly-mean temperature histograms. Figure 10 shows the annual variation of the histograms at the North Pole and $p=2.6 \mathrm{hPa}$ level. If this figure is compared with the observed re- sults shown in Fig. 2, the merit of the numerical experimentation is evident; this longer dataset gives much clear form of the frequency distributions. Internally generated interannual variation is large in spring (largest in March) in the run of $h_{0}=500 \mathrm{~m}$, while it is very large during winter (largest in January) in the run of $h_{0}=1000 \mathrm{~m}$. The distributions for $h_{0}=500 \mathrm{~m}$ have positive skewness for a longer months from autumn to spring and extremely large skewness is found in March. On the other hand, those for $h_{0}=1000 \mathrm{~m}$ are positively skewed in autumn and bimodal in winter. The bimodal structure might be related with two possible seasonal evolutions in winter found by Scott and Haynes (2002), although the intraseasonal variation is much complicated (Taguchi and Yoden 2002b). A possible application of a nonlinear dynamical perspective to the present system with bimodal frequency distributions will be discussed in the following section. If the real atmosphere has similar frequency distribution to the present result, the usefulness of 

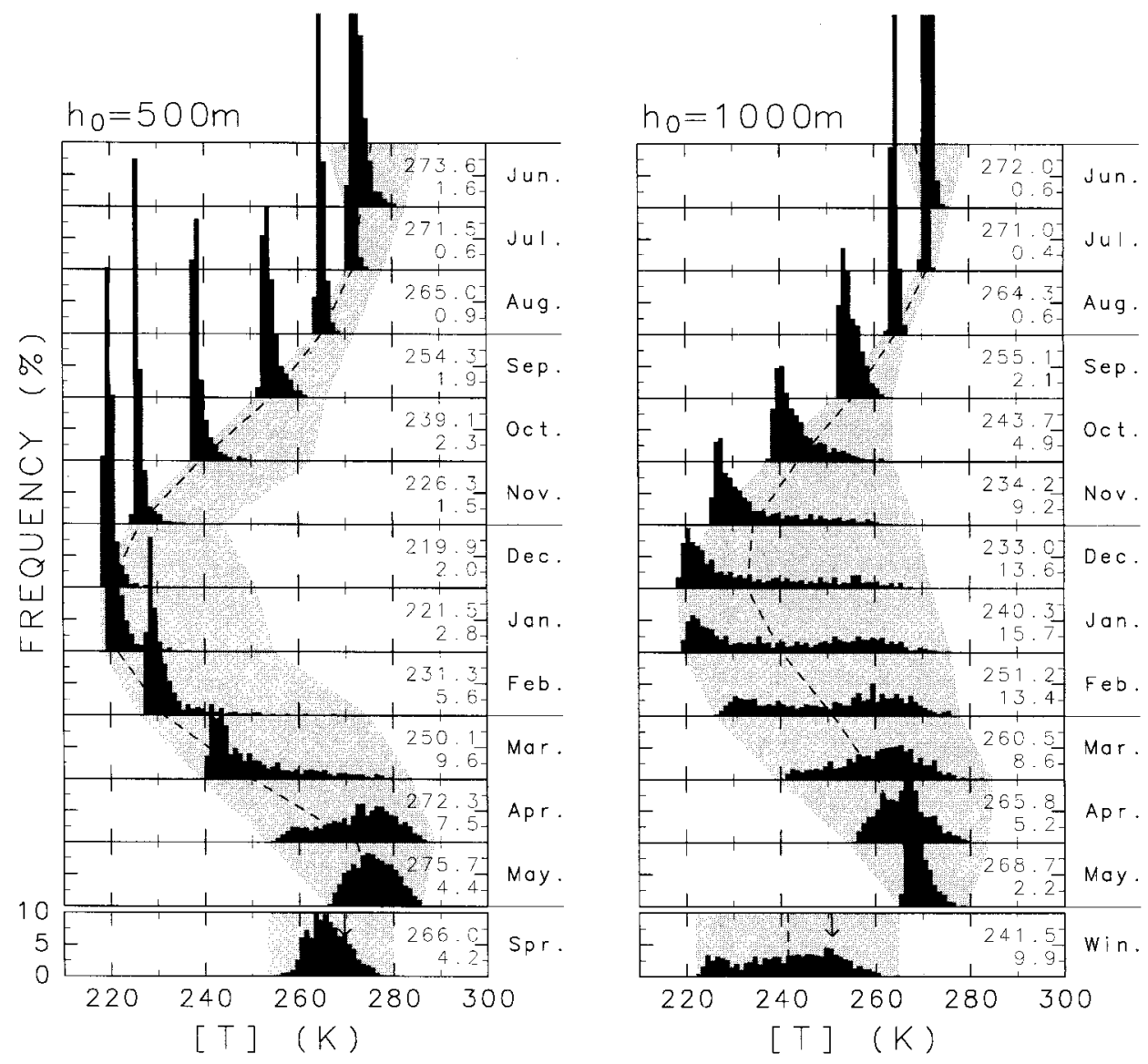

Fig. 10. Frequency distributions of the monthly mean temperature at $86^{\circ} \mathrm{N}$ and $2.6 \mathrm{hPa}$ for the two millennium integrations: $h_{0}=500 \mathrm{~m}$ (left) and $1000 \mathrm{~m}$ (right). Dashed line denotes the 1000-year mean annual variation of the monthly mean temperature, and shade shows the variable range. Averages and standard deviations for the 1000-year data are also written on the right hand side of each panel (top and bottom numbers, respectively). Frequency distributions for a seasonal mean are also displayed in the bottom: spring mean for $h_{0}=500 \mathrm{~m}$ and winter mean for $h_{0}=1000 \mathrm{~m}$. The downward arrow in the seasonal mean indicates a threshold value for the 200 years of highest temperature.

ordinary statistical significance tests, assuming a Gaussian distribution, might be questioned.

\section{Concluding remarks}

Generally, parameter sweep experiments are important to investigate nonlinear systems because of the limitation of linear interpolation in parameter space. Such experiments are useful for understanding the dynamical mechanism. Recently, it became possible to use an MCM with $\mathrm{O}\left(10^{5}\right)$ degrees of freedom for a parameter sweep experiment to study the internal variations of the T-S coupled system in mid- and high-latitudes (Taguchi et al. 2001; Taguchi and Yoden 2002a,b,c). The coupling process is fundamentally a two-way interaction between the troposphere and stratosphere in which planetary waves play an important role. In the realistic range of topographic amplitude $h_{0}$, the generation of planetary waves is a highly nonlinear process due to the interactions with the mean zonal flow and baroclinic disturbances. Thus, the traditional linear ideas for the generation are of limited use when they are applied to the internal variations under consideration.

Internal intraseasonal and interannual variations we obtained are large in the dynami- 
cally active season, that is, in spring for small $h_{0}$ but in winter for large $h_{0}$ (Figs. 9 and 10). These variations show some similar characteristics of the real atmosphere (Figs. 1 and 2), although no interannual variation of the external forcings is permitted in this model. The internal variations in this T-S coupled system will be understood more deeply by sophisticated analyses with the dynamical methods based on the wave-mean flow interaction theory in our future work.

It is also interesting to ask the relative importance of small-amplitude external forcings, such as the solar cycle, eruptions of volcanos, and so on as stated in Section 2, in the interannual variations of the real atmosphere. Large internal variability and a clear bimodality seen in the frequency distributions of the monthlymean polar temperature in late winter (Fig. 10) remind us that a nonlinear dynamical perspective is likely to be necessary to appreciate the effects of small-amplitude external forcings.

First, we should be very careful to draw any conclusion from data with limited length, either from a numerical experiment or real observation, because the large internal variability may produce spurious "response" due just to the limited size of the sample. Longer datasets would give more statistically reliable conclusions, although it might be very difficult to obtain such datasets. Our millennium integrations show that a dataset of one hundred years is enough to obtain reliable values of the time mean, and that a dataset of several hundred years is enough for the standard deviation, but much longer data are necessary to obtain reliable values of higher moments such as the skewness.

Much longer datasets are quite desirable, but we have no intention of insisting that detecting the effects of small-amplitude external forcings is hopeless. For example, the bimodality in the frequency distributions gives a hint of the possibility of stochastic resonance (e.g., Wiesenfeld and Moss 1995; Moss and Wiesenfeld 1995). Benzi et al. (1982) introduced a concept of stochastic resonance in a system with bimodality to explain the glacial-interglacial cycles in paleoclimatic records; if relatively short-term fluctuations (noise) in the system have enough intensity as an internal stochastic forcing, a small-amplitude periodicity in the external forcing could be greatly amplified by regular transitions between the bimodal states. If we could regard baroclinic disturbances in the troposphere as a source of such stochastic forcing, small changes in a bistable energy potential which produces the bimodality might be amplified by the stochastic resonance.

Palmer $(1998,1999)$ gave another example which shows the importance of nonlinearity of the system to appreciate the effects of smallamplitude external forcings. Again the existence of bimodality (or, multiple metastable states) is the key point. He demonstrated with an LOM that the response to a small-amplitude external forcing is primarily changes in the residence frequency associated with the metastable states. The external forcing does not have much influence on the structure of metastable states. If this concept is applicable to our T-S coupled system, perturbation runs with small changes in an external forcing would give significant changes in the frequency distributions of the monthly-mean temperature as shown in Fig. 10. For example, a series of parameter sweep experiments can be done to investigate the QBO effect by changing the configuration of the mean zonal wind in the equatorial stratosphere.

In conclusion, it is important to notice that the T-S coupled system is a typical nonlinear system with large internal variability and nonGaussian frequency distributions. We should be careful not to misuse linear concepts to analyze the data obtained from such system.

\section{Acknowledgments}

GFD-DENNOU Library was used for drawing the figures (SGKS Group 1995). This work was supported in part by the Grant-in-Aid for Scientific Research "Stratospheric Variations and Climate" of the Ministry of Education, Culture, Sports, Science and Technology of Japan, and by the Grant-in-Aid for the Research for the Future Program "Computational Science and Engineering" of the Japan Society for the Promotion of Science.

\section{References}

Andrews, D.G., 2000: An Introduction to Atmospheric Physics. Cambridge University Press, 229pp. , J.R. Holton and C.B. Leovy, 1987: Middle Atmosphere Dynamics. Academic Press, 489pp. 
Baldwin, M.P. and T.J. Dunkerton, 1999: Propagation of the Arctic Oscillation from the stratosphere to the troposphere. J. Geophys. Res., 104, 30937-30946.

and - 2001: Stratospheric harbingers of anomalous weather regimes. Science, 294, 581-584.

, L.J. Gray, T.J. Dunkerton, K. Hamilton, P.H. Haynes, W.J. Randel, J.R. Holton, M.J. Alexander, I. Hirota, T. Horinouchi, D.B.A. Jones, J.S. Kinnersley, C. Marquardt, K. Sato and M. Takahashi, 2001: The quasi-biennial oscillation. Rev. of Geophys., 39, 179-229.

Benzi, R., G. Parisi, A. Sutera and A. Vulpiani, 1982: Stochastic resonance in climatic change. Tellus, 34, 10-16.

Black, R.X. 2002: Stratospheric forcing of surface climate in the Arctic oscillation. J. Climate, 15, 268-277.

Blackmon, M., B. Boville, F. Bryan, R. Dickinson, P. Gent, J. Kiehl, R. Moritz, D. Randall, J. Shukla, S. Solomon, G. Bonan, S. Doney, I. Fung, J. Hack, E. Hunke, J. Hurrell, J. Kutzbach, J. Meehl, B. Otto-Bliesner, R. Saravanan, E.K. Schneider, L. Sloan, M. Spall, K. Taylor, J. Tribbia and W. Washington, 2001: The Community Climate System Model. Bull. Amer. Meteor. Soc., 82, 2357-2376.

Boville, B.A., 1984: The influence of the polar night jet on the tropospheric circulation in a GCM. $J$. Atmos. Sci., 41, 1132-1142.

Chao, W.C., 1985: Sudden stratospheric warmings as catastrophes. J. Atmos. Sci., 42, 1631-1646.

Chen, M., C.R. Mechoso and J.D. Farrara, 2001: Interannual variations in the stratospheric circulation with a perfectly steady troposphere. $J$. Geophys. Res., 106, 5161-5172.

Chen, P. and W. Robinson, 1992: Propagation of planetary waves between the troposphere and stratosphere. J. Atmos. Sci., 49, 2533-2545.

Christiansen, B., 1999: Stratospheric vacillations in a general circulation model. J. Atmos. Sci., 56, 1858-1872.

, 2000a: Chaos, quasiperiodicity, and interannual variability: Studies of a stratospheric vacillation model. J. Atmos. Sci., 57, 31613173 .

, 2000b: A model study of the dynamical connection between the Arctic Oscillation and stratospheric vacillations. J. Geophys. Res., 105, 29461-29474.

, 2001: Downward propagation of zonal mean zonal wind anomalies from the stratosphere to the troposphere: Model and reanalysis. J. Geophys. Res., 106, 27307-27322.

Dunkerton, T.J., 1991: Nonlinear propagation of zonal winds in an atmosphere with Newtonian cooling and equatorial wavedriving. J. Atmos. Sci., 48, 236-263.

Gray, L.J., E.F. Drysdale, T.J. Dunkerton and B.N. Lawrence, 2001: Model studies of the interannual variability of the northern-hemisphere stratospheric winter circulation: The role of the quasi-biennial oscillation. Quart. J. Roy. Meteor. Soc., 127, 1413-1432.

—, S. Sparrow, M. Juckes, A. O'Neill and D.G. Andrews, 2002: Model studies of winter hemisphere stratospheric flow regimes. Submitted to Quart. J. Roy. Meteor. Soc.

Hamilton, K., 1998: Effects of an imposed quasibiennial oscillation in a comprehensive troposphere-stratosphere-mesosphere general circulation model. J. Atmos. Sci., 55, 23932418.

Hartley, D.E., J.T. Villarin, R.X. Black and C.A. Davis, 1998: A new perspective on the dynamical link between the stratosphere and troposphere. Nature, 391, 471-474.

Hartmann, D.L. and F. Lo, 1998: Wave-driven zonal flow vacillation in the Southern Hemisphere. $J$. Atmos. Sci., 55, 1303-1315.

- J.M. Wallace, V. Limpasuvan, D.W.J. Thompson and J.R. Holton, 2000: Can ozone depletion and global warming interact to produce rapid climate change? PNAS, 97, 14121417.

Haynes, P.H., C.J. Marks, M.E. McIntyre, T.G. Shepherd and K.P. Shine, 1991: On the "downward control" of extratropical diabatic circulations by eddy-induced mean zonal forces. $J$. Atmos. Sci., 48, 651-678.

and T.G. Shepherd, 1989: The importance of surface pressure changes in the response of the atmosphere to zonally-symmetric thermal and mechanical forcing. Quart. J. Roy. Meteor. Soc., 115, 1181-1208.

Held, I.M., 1983: Stationary and quasi-stationary eddies in the extratropical troposphere: theory. In Large-Scale Dynamical Processes in the Atmosphere edited by B.J. Hoskins and R.P. Pearce. Academic Press, 127-168.

Hide, R. and P.J. Mason, 1975: Sloping convection in a rotating fluid. Adv. Phys., 24, 47-100.

Holton, J.R. and J. Austin, 1991: The influence of the equatorial $\mathrm{QBO}$ on sudden stratospheric warmings. J. Atmos. Sci., 48, 607-618.

- , P.H. Haynes, M.E. McIntyre, A.R. Douglass, R.B. Rood and L. Pfister, 1995: Stratospheretroposphere exchange. Rev. Geophys., 33, 403439 .

and C. Mass, 1976: Stratospheric vacillation cycles. J. Atmos. Sci., 33, 2218-2225.

and H.-C. Tan, 1980: The influence of the equatorial quasi-biennial oscillation on the 
global circulation at $50 \mathrm{mb}$. J. Atmos. Sci., 37, 2200-2208.

and - 1982: The quasi-biennial oscillation in the Northern Hemisphere lower stratosphere. J. Meteor. Soc. Japan, 60, 140148.

- and W.M. Wherbein, 1980: The role of forced planetary waves in the annual cycle of the zonal mean circulation of the middle atmosphere. J. Atmos. Sci., 37, 1968-1983.

and 1981: A further study of the annual cycle of the zonal mean circulation in the middle atmosphere. J. Atmos. Sci., 38, 15041509.

Horinouchi, T. and S. Yoden, 1998: Wave-mean flow interaction associated with a QBO-like oscillation simulated in a simplified GCM. J. Atmos. Sci., 55, 502-526.

Hoskins, B.J., 1983: Dynamical processes in the atmosphere and the use of models. Quart. J. Roy. Meteor. Soc., 109, 1-21.

-, M.E. McIntyre and A.W. Robertson, 1985: On the use and significance of isentropic potential vorticity maps. Quart. J. Roy. Meteor. Soc., 111, 877-946.

Kitoh, A., H. Koide, K. Kodera, S. Yukimoto and A. Noda, 1996: Interannual variability in the stratospheric-tropospheric circulation in a coupled ocean-atmosphere GCM. Geophys. Res. Lett., 23, 543-546.

Kodera, K. and M. Chiba, 1995: Tropospheric circulation changes associated with stratospheric sudden warmings: A case study. J. Geophys. Res., 100, 11055-11068.

and Y. Kuroda, 2000a: Tropospheric and stratospheric aspects of the Arctic Oscillation. Geophys. Res. Lett., 27, 3349-3352.

- and 2000b: A mechanistic model study of slowly propagating coupled stratospheretroposphere variability. J. Geophys. Res., 105, 12361-12370.

Kuroda Y. and K. Kodera, 1999: Role of planetary waves in the stratosphere-troposphere coupled variability in the northern hemisphere winter. Geophys. Res. Lett., 26, 2375-2378. and - 2001: Variability of the polar night jet in the Northern and Southern Hemispheres. J. Geophys. Res., 106, 20703-20713.

Labitzke, K.G. and H. van Loon, 1999: The stratosphere: Phenomena, history, and relevance. Springer, 179pp.

Lahoz, W.A., 2000: Northern hemisphere winter stratosphere variability in The Met. Office Unified Model. Quart. J. Roy. Meteor. Soc., 126, 2605-2630.

Lau, N.-C., 1992: Climate variability simulated in GCMs. In Climate System Modeling edited by
K.E. Trenberth. Cambridge University Press, 617-642.

Limpasuvan, V. and D.L. Hartmann, 1999: Eddies and the annular modes of climate variability. Geophys. Res. Lett., 26, 3133-3136.

- and - 2000: Wave-maintained annular modes of climate variability. J. Climate, 13, 4414-4429.

Lorenz, E.N., 1963: The mechanics of vacillation. J. Atmos. Sci., 20, 448-464.

Matsuno, T., 1971: A dynamical model of the stratospheric sudden warming. J. Atmos. Sci., 28, 1479-1494.

Moss, F. and K. Wiesenfeld, 1995: The benefits of background noise. Sci. Amer., 273-2, 66-69.

Niwano, M. and M. Takahashi, 1998: The influence of the equatorial QBO on the Northern Hemisphere winter circulation of a GCM. J. Meteor. Soc. Japan, 76, 453-461.

O'Sullivan, D. and T.J. Dunkerton, 1994: Seasonal development of the extratropical QBO in a numerical model of the middle atmosphere. $J$. Atmos. Sci., 51, 3706-3721.

and R.E. Young, 1992: Modeling the quasibiennial oscillation's effect on the winter stratospheric circulation. J. Atmos. Sci., 49, 24372448.

Palmer, T.N., 1998: Nonlinear dynamics and climate change: Rossby's legacy. Bull. Amer. Meteor. Soc., 79, 1411-1423.

, 1999: A nonlinear dynamical perspective on climate prediction. J. Climate, 12, 575591.

Pawson, S., K. Kodera, K. Hamilton, T.G. Shepherd, S.R. Beagley, B.A. Boville, J.D. Farrara, T.D.A. Fairlie, A. Kitoh, W.A. Lahoz, U. Langematz, E. Manzini, D.H. Rind, A.A. Scaife, K. Shibata, P. Simon, R. Swinbank, L. Takacs, R.J. Wilson, J.A. Al-Saadi, M. Amodei, M. Chiba, L. Coy, J. de Grandpre, R.S. Eckman, M. Fiorino, W.L. Grose, H. Koide, J.N. Koshyk, D. Li, J. Lerner, J.D. Mahlman, N.A. McFarlane, C.R. Mechoso, A. Molod, A. O'Neill, R.B. Pierce, W.J. Randel, R.B. Rood and F. Wu, 2000: The GCM-reality inter-comparison project for SPARC (GRIPS): Scientific issues and initial results. Bull. Amer. Meteor. Soc., 81, 781-796.

- A. Meyer and S. Leder, 1995: Internal variability in a perpetual January integration of a troposphere-stratosphere-mesosphere GCM. Quart. J. Roy. Meteor. Soc., 121, 369-397.

Philander, S.G.H., 1990: El Niño, La Niña, and the southern oscillation. Academic Press, 293pp.

Plumb, R.A., 1981: Instability of the distorted polar night vortex: A theory of stratospheric warmings. J. Atmos. Sci., 38, 2514-2531. , 1989: On the seasonal cycle of stratospheric 
planetary waves. Pure Appl. Geophys., 130, 233-242.

Ramaswamy, V., M.-L. Chanin, J. Angell, J. Barnett, D. Gaffen, M. Gelman, P. Keckhut, Y. Koshelkov, K. Labitzke, J.-J.R. Lin, A. O’Neill, J. Nash, W. Randel, R. Rood, K. Shine, M. Shiotani and R. Swinbank, 2001: Stratospheric temperature trends: Observations and model simulations. Rev. Geophys., 39, 71-122.

Randall, D.A. (Ed.), 2000: General circulation model development. Academic Press, 803pp.

Randel, W.J. and P.A. Newman, 1998: The stratosphere in the Southern Hemisphere. In Meteorology of the Southern Hemisphere edited by D.J. Karoly and D.G. Vincent. Meteor. Monographs, 27, 243-282.

Scaife, A.A. and I.N. James, 2000: Response of the stratosphere to interannual variability of tropospheric planetary waves. Quart. J. Roy. Meteor. Soc., 126, 275-297.

Scott, R.K. and P.H. Haynes, 1998: Internal interannual variability of the extratropical stratospheric circulation: The low-latitude flywheel. Quart. J. Roy. Meteor. Soc., 124, 2149-2173. and - 2000: Internal vacillations in stratosphere-only models. J. Atmos. Sci., 57, 3233-3250.

— and 2002: The seasonal cycle of planetary waves in the winter stratosphere. $J$. Atmos. Sci., 59, 803-822.

SGKS Group, 1995: DCL-5.0., http://www.gfddennou.org/library/dcl/, GFD-Dennou Club (documents are in Japanese).

Shepherd, T.G., 2000: The middle atmosphere. J. Atmos. Solar-Ter. Phys., 62, 1587-1601.

Shindell, D.T., R.L. Miller, G.A. Schmidt and L. Pandolfo, 1999a: Simulation of recent northern winter climate trends by greenhouse-gas forcing. Nature, 399, 452-455.

, D. Rind, N. Balachandran, J. Lean and P. Lonergan, 1999b: Solar cycle variability, ozone, and climate. Science, 284, 305-308.

Taguchi, M., T. Yamaga and S. Yoden, 2001: Internal variability of the troposphere-stratosphere coupled system simulated in a simple global circulation model. J. Atmos. Sci., 58, 31843203.

- and S. Yoden, 2002a: Internal interannual variability of the troposphere-stratosphere coupled system in a simple global circulation model. Part I: Parameter sweep experiment. J. Atmos. Sci., in press.

- and - 2002b: Internal intraseasonal and interannual variations of the tropospherestratosphere coupled system in a simple global circulation model. Part II: Millennium integrations. J. Atmos. Sci., in press.

- and - 2002c: A parameter-sweep experiment on the annular variability with a simple global circulation model. J. Meteor. Soc. Japan, 80, 1075-1086.

Thompson, D.W.J. and J.M. Wallace, 1998: The Arctic Oscillation signature in the wintertime geopotential and temperature fields. Geophys. Res. Lett., 25, 1297-1300.

and 2000: Annular modes in the extratropical circulation. Part I: month-to-month variability. J. Climate, 13, 1000-1016.

Trenberth, K.E. (Ed.), 1992: Climate system modeling. Cambridge University Press, 788pp.

Wakata, Y. and M. Uryu, 1987: Stratospheric multiple equilibria and seasonal variations. J. Meteor. Soc. Japan, 65, 27-42.

Wiesenfeld, K. and F. Moss, 1995: Stochastic resonance and the benefits of noise: from ice ages to crayfish and SQUIDs. Nature, 373, 3336.

WMO, 1998: Stratospheric processes and their role in climate (SPARC) implementation plan. WCRP-105, WMO/TD-NO. 914, 131pp.

Yamazaki, K. and Y. Shinya, 1999: Analysis of the Arctic Oscillation simulated by AGCM. J. Meteor. Soc. Japan, 77, 1287-1298.

Yoden, S., 1987a: Bifurcation properties of a stratospheric vacillation model. J. Atmos. Sci., 44, 1723-1733.

, 1987b: A new class of stratospheric vacillations in a highly truncated model due to wave interference. J. Atmos. Sci., 44, 3696-3709.

, 1990: An illustrative model of seasonal and interannual variations of the stratospheric circulation. J. Atmos. Sci., 47, 1845-1853.

, 1997: Classification of simple low-order models in geophysical fluid dynamics and climate dynamics. Nonlinear Analysis, Theory, Methods \& Applications, 30, 4607-4618. , Y. Naito and S. Pawson, 1996: A further analysis of internal variability in a perpetual January integration of a tropospherestratosphere-mesosphere GCM. J. Meteor. Soc. Japan, 74, 175-188.

, T. Yamaga, S. Pawson and U. Langematz, 1999: A composite analysis of the stratospheric sudden warmings simulated in a perpetual January integration of the Berlin TSM GCM. J. Meteor. Soc. Japan, 77, 431-445.

Yulaeva, E., J.R. Holton and J.M. Wallace, 1994: On the cause of the annual cycle in the tropical lower-stratospheric temperatures. J. Atmos. Sci., 51, 169-174. 